\title{
B-cell activating factor BAFF as a novel alert marker for the immunological risk stratification after kidney transplantation
}

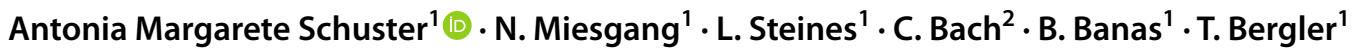

Received: 7 December 2020 / Accepted: 18 May 2021 / Published online: 10 August 2021

(c) The Author(s) 2021

\begin{abstract}
The B cell activating factor BAFF has gained importance in the context of kidney transplantation due to its role in B cell survival. Studies have shown that BAFF correlates with an increased incidence of antibody-mediated rejection and the development of donor-specific antibodies. In this study, we analyzed a defined cohort of kidney transplant recipients who were treated with standardized immunosuppressive regimens according to their immunological risk profile. The aim was to add BAFF as an awareness marker in the course after transplantation to consider patient's individual immunological risk profile. Included patients were transplanted between 2016 and 2018. Baseline data, graft function, the occurrence of rejection episodes, signs of microvascular infiltration, and DSA kinetics were recorded over 3 years. BAFF levels were determined 14 d, 3 and 12 months post transplantation. Although no difference in graft function could be observed, medium-risk patients showed a clear dynamic in their BAFF levels with low levels shortly after transplantation and an increase in values of $123 \%$ over the course of 1 year. Patients with high BAFF values were more susceptible to rejection, especially antibody-mediated rejection and displayed intensified microvascular inflammation; the combination of high BAFF+DSA puts patients at risk. The changing BAFF kinetics of the medium risk group as well as the increased occurrence of rejections at high BAFF values enables BAFF to be seen as an awareness factor. To compensate the changing immunological risk, a switch from a weaker induction therapy to an intensified maintenance therapy is required.
\end{abstract}

Keywords BAFF $\cdot$ ABMR $\cdot$ Immunological risk stratification $\cdot$ Kidney transplantation

\section{Introduction}

The B cell activating factor BAFF plays a crucial role in the development and survival of B-lymphocytes. Because of this role, BAFF gained attention in recent years, also in the context of kidney transplantation. B-lymphocytes make a decisive contribution to the development of donor-specific antibodies through their regulatory functions such as cytokine production, but especially through their conversion into antibody-producing cells [1]. They are therefore of central importance in the context of rejection, mainly antibodymediated rejection, which is a major cause of renal allograft dysfunction and subsequent graft loss [2, 3].

Antonia Margarete Schuster

antonia-margarete.schuster@ukr.de

1 Department of Nephrology, University Hospital Regensburg, Franz-Josef-Strauß-Allee 11, 93053 Regensburg, Germany

2 Department of Internal Medicine 5, University Hospital Erlangen, Erlangen, Germany
BAFF, also known as BLyS (B lymphocyte stimulator), TALL1 (TNF and apoL-related leukocyte- overexpressed ligand 1), or TNFSF13B (TNF superfamily member 13B) belongs to the TNF superfamily [4] and could be detected in both a soluble and a membrane-bound form.

Different cells express BAFF, including neutrophils, monocytes, macrophages, and dendritic cells. IL-10, IFN$\gamma$, and IFN- $\alpha$ are considered a stimulus of BAFF expression, whereas IL-4 tends to inhibit BAFF expression [5]. BAFF attaches to three known receptors, transmembrane activator and CAML interactor (TACI), B cell maturation antigen (BCMA), and BAFF receptor (BAFF-R or BR3), which are expressed at different times in $\mathrm{B}$ cell development and proliferation [6].

By stimulating pro-survival oncogenes like Bcl-2, BAFF is crucial for the survival and proliferation of B-lymphocytes. In mouse models it was shown that the development of mature B lymphocytes in particular is dependent on BAFF and that without BAFF stimulation there is a rapid loss of B cells $[6,7]$. 
Also in the context of autoimmune diseases such as lupus erythematosus and Sjogren's syndrome, it is assumed that BAFF is relevant due to overexpression and thereby formation of autoreactive B cells [7], and since 2011 belimumab, a humanized monoclonal antibody against BAFF was approved [8].

In the context of transplantation, there are still inconsistent data concerning the relevance of BAFF. In their study, Thibault-Espitia et al. were able to show that increased BAFF levels after transplantation are associated with an increased occurrence of de novo donor-specific antibodies and graft dysfunction [9]. However, this could not be reproduced in other studies. In a previous work, we were able to show that BAFF reflects the immunization status (no PRA vs. verifiable PRA) of patients before transplantation and that patients with higher BAFF values in the followup showed an increased risk for an impaired graft function [10]. It was also demonstrated that increased BAFF levels go hand in hand with an increased occurrence of rejections, especially antibody-mediated rejections [11]. In an experimental KTx model in rats, our group showed that chronic underdosing of immunosuppression - analogous to the non-adherence of transplanted patients - induced expression of BAFF and BAFF receptor within allografts [12] and that when a monoclonal anti-BAFF antibody was applied in the that rodent KTx model, DSA development was partially inhibited [13].

BAFF has been used as a therapeutic target in a phase 2a study. Usage of the BAFF inhibitor tabalumab in preimmunized patients with end-stage renal disease resulted in a reduction of the cPRA levels [14]. In a more clinically relevant setting, Banham et al. conducted a double-blind, randomized, placebo-controlled phase 2 study in which kidney transplant patients were treated with an anti-BAFF antibody, belimumab, or placebo after transplantation. The aim of the study was a more detailed analysis of the influence of belimumab on $\operatorname{IgG}$ production and on B cell homeostasis. Despite similar rates of adverse events in both groups, the co-primary endpoint of a reduced naïve B-cell amount was not achieved [15].

The aim of our current study was to analyze a pre-defined highly standardized cohort of kidney transplant patients, who were treated with a specific immunosuppressive regimen according to their immunological risk profile, with regard to the development of BAFF levels. The immunological risk profile of kidney transplant recipients is currently defined by clinical parameters such as PRA level, presence of DSA, development of de novo DSA, and re-transplantation and determines the choice of immunosuppressive induction and maintenance therapy. This is also reflected in the current KDIGO guidelines. Here, the patients are stratified into a low, medium, and high-risk group based on immunology, and both induction and maintenance therapy are then adapted accordingly. Apart from such clinical factors, functional $\mathrm{T}$ cell assays, such as a specific ELISpot testing, in the context of CMV infections, are available. However, the possibility of a reliable B cell monitoring is currently not established on a routine basis. Given the important functions of B cells [1], this is especially necessary in the transplant setting. Based on previous experience with BAFF, it may be possible to further develop BAFF as an immunological alert marker in the course of kidney transplantation with the aim of administering a tailored immunosuppression for each patient.

Besides monitoring BAFF in the follow-up in correlation to recipients histopathological graft lesions, the occurrence of rejection episodes (TCMR, AMR) and the development of de novo donor-specific antibodies were examined.

\section{Material and methods}

\section{Patients' baseline characteristics}

Initially, all patients who were transplanted at our center from January 1st, 2016, to December 31th, 2018, were identified $(n=122)$. Based on their immunological risk profile, the patients were divided into three, pre-defined groups before transplantation: low, medium, or high immunological risk. Both induction therapy and maintenance immunosuppression were then aligned according to this risk stratification. A low immunological risk was defined as an $\mathrm{AB} 0$ compatible first transplant, a CDC-PRA level of less than 5\% and no detectable anti-HLA- (HLA-A;-B;-C;-DR;-DP;DQ) or donor-specific antibodies. Patients with a CDC-PRA level of 5-30\% and/or detectable anti-HLA antibodies in the absence of donor-specific antibodies or patients with a previous transplant but without immunological graft loss within 2 years were stratified into the medium risk group. Once any donor-specific antibodies could be detected (MFI > 500) or any CDC-PRA level above $30 \%$ and patients have suffered from an early immunological graft loss in a previous transplant, they were assigned to the high risk group (Supplement Table 1). The sum of HLA-mismatches was not taken into account when classifying the immunological risk. In our cohort, there were 119 Caucasian $(97.5 \%)$ and 3 patients of non-Caucasian origin (2.5\%). Because of this fact, race was not taken into account in the distribution of the immunological risk. According to the German transplantation law, a donation after cardiac death (DCD) is not permitted, so that all post-mortem donations were made through donations after brain death (DBD). Out of the 122 transplants performed, 41 were from a living donation (33.6\%) from which 17 were from blood relatives $(41.5 \%)$. Forty-nine patients received an organ from a donor with extended donor criteria (ECD) $(40.2 \%$ ). 
A total of 122 patients were enrolled, with 44 patients in the low immunological risk group (36.1\%), 34 patients in the medium immunological risk group (27.9\%), and 44 patients in the high immunological risk group (36.1\%). Lowor medium-risk patients received induction therapy with a CD 25 monoclonal antibody basiliximab (Novartis), a therapy that does not deplete $\mathrm{T}$ cells but has an immunomodulatory effect. In contrast, high-risk patients were induced with thymoglobulin in a routinely used dose $(6 \mathrm{mg} / \mathrm{kg}$ body weight) (Sanofi). The maintenance immunosuppressive therapy was in all three groups based on a calcineurin inhibitor (tacrolimus) in combination with a proliferation inhibitor (mycophenolat mofetil or mycophenolat acid) and steroids.

Depending on the pre-defined risk profile, the respective medication was reduced in the follow-up after transplantation. Depending on the underlying renal disease, steroids were stopped after three months or maintained at a dose of $5 \mathrm{mg}$. The tacrolimus target levels were the same among the three groups. Shortly after transplantation, a target level of 12 was aimed for and was reduced to $8-10 \mu \mathrm{g} / \mathrm{l}$ by the end of month 3 . The target level for months 4-12 was $6-8 \mu \mathrm{g} / \mathrm{l}$ and after month 12 a target level of $4-6 \mu \mathrm{g} / \mathrm{l}$ was maintained. The differences in the immunosuppressive regime were determined on the one hand by the induction therapy and on the other hand by the dosages in mycophenolate acid and steroids. The exact details between the immunosuppressive regimens can be found in the supplement in Table 2 .

At our center, post-transplant monitoring is carried out according to a predefined standard, as part of the so-called Regensburger Transplantationsnachsorge. Furthermore, all relevant transplantrelated data and donor data are archived. Human tissue was analyzed according to the approval of the Ethics Committee of the Medical Faculty of the University of Regensburg.

In addition to the analysis of the baseline data, clinical endpoints such as graft function represented by creatinine, eGFR, and albuminuria over an observation period of 3 years and dosage of the immunosuppression were recorded. The BAFF levels of all patients at 14 days, 3 months, and 12 months were then determined by ELISA.

\section{BAFF-ELISA analysis}

BAFF levels in patients' sera were measured by using the human Baff/BlyS/TNFSF13 B immunoassay (R\&D Systems, Minneapolis, USA) according to the manufacturer's recommendations. The minimum detectable concentration was $62.5 \mathrm{pg} / \mathrm{ml}$ and OD-measurement was done with a Tecan reader (Männedorf, Switzerland).

\section{Histopathological analysis}

Furthermore, we analyzed allograft biopsies (protocol biopsy $14 \mathrm{~d}$ and 3 months, as well as indication biopsies during the observation period) with regard to rejection episodes according to the BANFF 2017 criteria's as well as in respect to allograft lesion score (tubulitis, peritubular capillaritis, etc.) [16]. Additionally, the de novo DSA kinetics ( 3 and 12 months postTx) were examined. All these parameters were compared first with the underlying immunological risk classification, secondly in regard to the BAFF median. The BAFF median at 14 days was calculated based on all BAFF values of the 122 patients at 14 days.

\section{Detection of de novo donor-specific antibodies}

Antibody tests were carried out according to the standards of the European Federation for Immunognetics (EFI). All patients were routinely tested for donor-specific antibodies prior to transplantation (preTxDSA) and at 3 and 12 months after transplantation (postTxDSA). Specifically, patients' sera were tested for presence/absence of anti-HLA antibodies using LABScreen ${ }^{\circledR}$ Mixed Assay (LSmixed, One Lambda, Inc, 22,801 Roscoe Blvd. West Hills, CA 91,304, USA), applying the manufacturer's recommended positive test ratio of $>2.2$. For positive sera, anti-HLA antibody specificity was determined using a single antigen assay for HLA class I (i.e., HLA$\mathrm{A} / \mathrm{B} / \mathrm{Cw}$ ) and/or HLA class II antigens (i.e., HLA-DR/DQ/DP; LABScreen ${ }^{\circledR}$ Single Antigen Assay, LS1A04 and LS2A01, One Lambda), according to the manufacturer's instructions. Positive results for antibody specificities in single antigen test were defined by a baseline normalized mean fluorescence intensity $(\mathrm{MFI})>500$. All antibody tests were analyzed on a LABScan 200® flow analyzer (One Lambda). Finally, donorspecific HLA-antibodies were determined via comparison of the assigned specificities with the donor HLA-type.

\section{Statistical analysis}

Continuous variables are presented as mean \pm standard deviation, whereas categorical data are shown as frequency distributions (n) and percentages (\%). A statistical analysis was performed by the Student's t-test with a $\mathrm{p}$ value $<0.05$ indicating a statistical significance. The analysis was calculated using Excel 2016.

\section{Results}

\section{Stratification in terms of the basic immunological risk}

\section{Patients' baseline characteristics}

As mentioned above, 122 patients were stratified into the three immunological risk groups (Table 1). No donor nor any recipient derived baseline marker differed between the groups. Regarding immunological aspects, patients in the 
low-risk group were noticed by significantly more HLA-B mismatches than the other two cohorts ( $\mathrm{p}=0.03$ resp. 0.04). According to the underlying stratification, patients with a high immunological risk showed significantly higher CDCPRA levels $(30 \%)$ than the low-risk $\left(0 \%, \mathrm{p}=1.7 \times 10^{-6}\right)$ and medium-risk group $(10 \% ; \mathrm{p}=0.008)$. Low-risk patients had a significantly shorter cold ischemia time (CIT) than medium-risk patients $(\mathrm{p}=0.04)$. There was no difference in terms of warm ischemia time (WIT). Detailed information concerning the baseline data is shown in Table 1 .

The follow-up time in the low-risk group was 20.5 months, in the medium group 22.3 months, and in the high-risk group 18.3 months on average.

\section{Immunosuppressive therapy}

Concerning the immunosuppression used, it is shown, as prescribed by the stratification, that the patients at high risk were induced significantly more frequently with thymoglobulin, whereas the other patients received basiliximab.

Fourteen days after transplantation, the high-risk patients had higher doses of mycophenolate acid than the two comparison groups. The level of significance was reached each time (low vs. high: $p=0.001$ and medium vs. high: $p=0.01$ ). Then, after 3 months, the high-risk population was treated with intensified doses of steroids ( $\mathrm{p}=0.03$ (vs. low risk) and $\mathrm{p}=0.009$ (vs. medium risk). No further differences
Table 1 Baseline characteristics and follow- up parameter of renal transplant recipients stratified for immunological risk for allograft rejection

\begin{tabular}{|c|c|c|c|}
\hline & Low risk $(n=44)$ & $\begin{array}{l}\text { Medium risk } \\
(\mathrm{n}=34)\end{array}$ & High risk $(n=44)$ \\
\hline Donor- age (years) & $54 \pm 15$ & $55 \pm 12$ & $51 \pm 19$ \\
\hline Donor — weight $(\mathrm{kg})$ & $77 \pm 18$ & $80 \pm 15$ & $78 \pm 22$ \\
\hline Donor- height $(\mathrm{cm})$ & $171 \pm 12$ & $174 \pm 9$ & $170 \pm 17$ \\
\hline Donor-sex (M:F) & $15: 29$ & $21: 13$ & $21: 23$ \\
\hline Recipient — age & $55 \pm 12$ & $50 \pm 13$ & $54 \pm 12$ \\
\hline Recipient- - weight (kg) & $76 \pm 10$ & $83 \pm 17$ & $77 \pm 16$ \\
\hline Recipient — height (cm) & $172 \pm 8$ & $174 \pm 9$ & $170 \pm 10$ \\
\hline Recipient — sex (M:F) & $33: 11$ & $23: 11$ & $29: 15$ \\
\hline $\operatorname{Re}-T x(n)$ & 2 & 0 & 6 \\
\hline Duration of RRT; years & $3.6 \pm 3.7$ & $5.1 \pm 4.0$ & $5.3 \pm 3.9$ \\
\hline \multicolumn{4}{|c|}{ Causes of end stage renal disease } \\
\hline ADPKD & 9 & 6 & 5 \\
\hline IgA- Nephropathy & 6 & 7 & 11 \\
\hline Hypertensive & 13 & 5 & 5 \\
\hline Diabetic & 4 & 2 & 5 \\
\hline Others & 12 & 14 & 18 \\
\hline \multicolumn{4}{|l|}{ HLA-mismatch } \\
\hline HLA-A & 0.9 & 0.8 & 0.7 \\
\hline HLA-B & $1.4^{\mathrm{a} ; \mathrm{b}}$ & $0.9^{\mathrm{a}}$ & $1^{\mathrm{b}}$ \\
\hline HLA-DR & 1.2 & 0.9 & 1 \\
\hline PRA $(\%)$ - current & $0^{\mathrm{b}}$ & $2^{\mathrm{c}}$ & $15^{\mathrm{b} ; \mathrm{c}}$ \\
\hline PRA (\%) — highest & $0^{\mathrm{a} ; \mathrm{b}}$ & $10^{\mathrm{a} ; \mathrm{c}}$ & $30^{\mathrm{b} ; \mathrm{c}}$ \\
\hline \multicolumn{4}{|l|}{ Ischemia time } \\
\hline CIT (h/min) & $6.5 / 29^{\mathrm{a}}$ & $7.8 / 21^{\mathrm{a}}$ & $8.5 / 27$ \\
\hline WIT (min) & 47 & 42 & 45 \\
\hline \multicolumn{4}{|l|}{ Rejection episodes (n) } \\
\hline TCMR & 6 & 0 & 7 \\
\hline AMR & 2 & 1 & 2 \\
\hline Borderline & 1 & 2 & 1 \\
\hline \multicolumn{4}{|c|}{ De novo donor specific antibodies } \\
\hline HLA class I (n/\%) & $0 / 0$ & $0 / 0$ & $6 / 13.6$ \\
\hline HLA class II (n/\%) & $2 / 4.5$ & $3 / 8.8$ & $5 / 11.4$ \\
\hline Graft loss (n/\%) & $3 / 6.8$ & $1 / 2.9$ & $1 / 2.3$ \\
\hline Death $(\mathrm{n} / \%)$ & $3 / 6.8$ & $0 / 0$ & $2 / 4.5$ \\
\hline
\end{tabular}

${ }^{\mathrm{a}}$ Low vs. medium: $\mathrm{p}<0.05$. ${ }^{\mathrm{b}}$ Low vs. high: $\mathrm{p}<0.05$, ${ }^{\mathrm{c}}$ medium vs. high: $\mathrm{p}<0.05$ 
in immunosuppressive therapy could be demonstrated. Detailed information concerning the immunosuppressive therapy is shown in Table 2 .

\section{Resulting allograft function}

With regard to the clinical course after transplantation, creatinine with the corresponding eGFR (CKD-EPI) and the albuminuria at the time points $14 \mathrm{~d}, 3$ and 12 months, and after 2 and 3 years were analyzed. There was no statistically significant difference detectable between the three groups at any of these time points (Supplement Table 3). In further analyzes, we could see that even in the stratification according to BAFF median, no influence on graft function (creatinine, eGFR, and albuminuria) could be found. On the other hand, analysis of influencing factors after transplantation (donor age, sum of HLA mismatches, and length of cold ischemia time) showed a deteriorated function both through donor age and duration of CIT.

\section{BAFF-ELISA analysis}

BAFF levels at $14 \mathrm{~d}, 3$ months, and 12 months after transplantation showed for patients with a low immunological risk profile initially a BAFF level of $459.9 \mathrm{pg} / \mathrm{ml} \pm 189.7 \mathrm{pg} / \mathrm{ml}$, which rose to $647.5 \mathrm{pg} / \mathrm{ml} \pm 353.1 \mathrm{pg} / \mathrm{ml}$ (3 months) and then to $767.9 \mathrm{pg} / \mathrm{ml} \pm 248.4 \mathrm{pg} / \mathrm{ml}$ (12 months). In the mediumrisk group, the initial value was $400.1 \mathrm{pg} / \mathrm{ml} \pm 212.1 \mathrm{pg} / \mathrm{ml}$ with an increase to $677.4 \mathrm{pg} / \mathrm{ml} \pm 329.0 \mathrm{pg} / \mathrm{ml}$ (3 months) and further to $890.7 \mathrm{pg} / \mathrm{ml} \pm 252.5 \mathrm{pg} / \mathrm{ml}$ (12 months). The BAFF starting level was $544.8 \mathrm{pg} / \mathrm{ml} \pm 251.9 \mathrm{pg} / \mathrm{ml}$ in the group with the high immunological risk profile and then changed to $828.5 \mathrm{pg} / \mathrm{ml} \pm 623.0 \mathrm{pg} / \mathrm{ml}$ (3 months) and $1018.8 \mathrm{pg} / \mathrm{ml} \pm 610.5 \mathrm{pg} / \mathrm{ml}$ (12 months) (Fig. 1). Initially the BAFF expression level of the medium-risk patients were similar to the low-risk patients ( $\mathrm{p}=0.01 \mathrm{vs}$. high risk). In the follow-up, medium-risk patients displayed the most intense increase in BAFF expression level (123\%) - which was even higher than in high-risk patients (87\%) and low risk
(67\%). Thus, after 12 months medium-risk patients were almost comparable to high-risk patients, which displayed a significant difference to low risk $(\mathrm{p}=0.04)$.

If one looked at the individual groups separately, it is noticeable that all patients from the medium risk group, who initially had BAFF values below the median, subsequently increased with their BAFF values after 3 months. No patient from this cohort showed BAFF values below the median at month 12. The situation is similar in the group with a high immunological risk. Only one patient was noticed with BAFF values below the median after 12 months.

\section{Histological findings}

All patients - even with a completely auspicious followup - received a protocol biopsy $14 \mathrm{~d}$ and 3 months after transplantation. Furthermore, indication biopsies were performed (e.g., for rise in creatinine, delayed graft function, new albuminuria, etc.). All biopsies were evaluated according to the BANFF 2017 criteria [16]. The results of the biopsies among the 3 groups and at different time points (0-30 d, 31-100 d, 101-365 d, > $365 \mathrm{~d}$ ) were compared regarding incidence of any rejection and chronic lesions. When rejections occurred, they were either classified as an acute $\mathrm{T}$ cell-mediated rejection or as an antibody-mediated rejection according to the BANFF criteria, which were then also addressed therapeutically. Subclinical rejections in the sense of borderline rejections did not lead to any change in therapy if kidney function was stable. Borderline rejections were found in all three groups (low risk, $\mathrm{n}=1$; medium risk, $\mathrm{n}=2$; high risk, $\mathrm{n}=1$ ).

A look at the biopsies with regard to the lesions scores showed that the medium-risk group had a statistically more significant occurrence of chronic lesions in the sense of interstitial fibrosis (ci) and interstitial atrophy (ct) compared to the low-risk group (ci: biopsies $<30$ days: $\mathrm{p}=1.1 \times 10^{-8}$; days 31-100: $\mathrm{p}=1.2 \times 10^{-15}$; biopsies up to day 365 : $\mathrm{p}=1.1 \times 10^{-09} ; \mathrm{ct}$ : biopsies $<30$ days: $\mathrm{p}=8.4 \times 10^{-8}$; 31-100 days: $p=7.1 \times 10^{-19}$; up to day $365: p=1.1 \times 10^{-9}$ ).

Table 2 Real life immunosuppressive doses and target levels of the three cohorts during follow-up until month 12 after transplantation

\begin{tabular}{|c|c|c|c|c|c|c|c|c|c|}
\hline & \multicolumn{3}{|c|}{ Low risk $(n=44)$} & \multicolumn{3}{|c|}{ Medium risk $(\mathrm{n}=34)$} & \multicolumn{3}{|c|}{ High risk $(n=44)$} \\
\hline & $\begin{array}{l}14 \text { days } \\
\text { postTX }\end{array}$ & $\begin{array}{l}3 \text { months } \\
\text { postTX }\end{array}$ & $\begin{array}{l}12 \text { months } \\
\text { post Tx }\end{array}$ & $\begin{array}{l}14 \text { days } \\
\text { postTX }\end{array}$ & $\begin{array}{l}3 \text { months } \\
\text { postTX }\end{array}$ & $\begin{array}{l}12 \text { months } \\
\text { post Tx }\end{array}$ & $\begin{array}{l}14 \text { days } \\
\text { postTX }\end{array}$ & $\begin{array}{l}3 \text { months } \\
\text { postTX }\end{array}$ & $\begin{array}{l}12 \text { months } \\
\text { post Tx }\end{array}$ \\
\hline $\begin{array}{l}\text { Calcineurin- } \\
\text { inhibitor }^{*}\end{array}$ & $11.3 \pm 2.9$ & $9.0 \pm 1.9$ & $6.7 \pm 2.2$ & $10.5 \pm 2.7$ & $9.1 \pm 2.8$ & $7.3 \pm 3.9$ & $10.7 \pm 2.5$ & $9.2 \pm 2.3$ & $7.0 \pm 2.1$ \\
\hline $\begin{array}{l}\text { Mycophenolat } \\
\text { acid(mg) }\end{array}$ & $1236.2 \pm 356.5^{\mathrm{b}}$ & $1046.7 \pm 429.7$ & $743.6 \pm 449.8^{2}$ & $1283.5 \pm 332.8$ & $1035.8 \pm 369$ & $971.4 \pm 382.5^{\mathrm{a}}$ & $1450 \pm 224^{b ; c}$ & $1202.5 \pm 396.8$ & $943.2 \pm 401.1$ \\
\hline $\begin{array}{l}\text { Steroids } \\
\text { (mg) }\end{array}$ & $14.7 \pm 4.1$ & $5.7 \pm 3.2^{b}$ & $2.9 \pm 5.5$ & $14.7 \pm 2.7$ & $5.8 \pm 1.6^{\mathrm{c}}$ & $3.2 \pm 2.3^{\mathrm{c}}$ & $16.2 \pm 4.1$ & $7.1 \pm 2.2^{\mathrm{b}: \mathrm{c}}$ & $4.5 \pm 1.6^{\mathrm{c}}$ \\
\hline
\end{tabular}

${ }^{*}$ The measured target levels are given here. ${ }^{\mathrm{a}}$ Low vs. medium: $\mathrm{p}<0.05$. ${ }^{\mathrm{b}}$ Low vs. high: $\mathrm{p}<0.05$, ${ }^{\mathrm{c}}$ medium vs. high: $\mathrm{p}<0.05$ 
Fig. 1 The BAFF level of the three groups over the observation period of 1 year. $\#=<0.05$

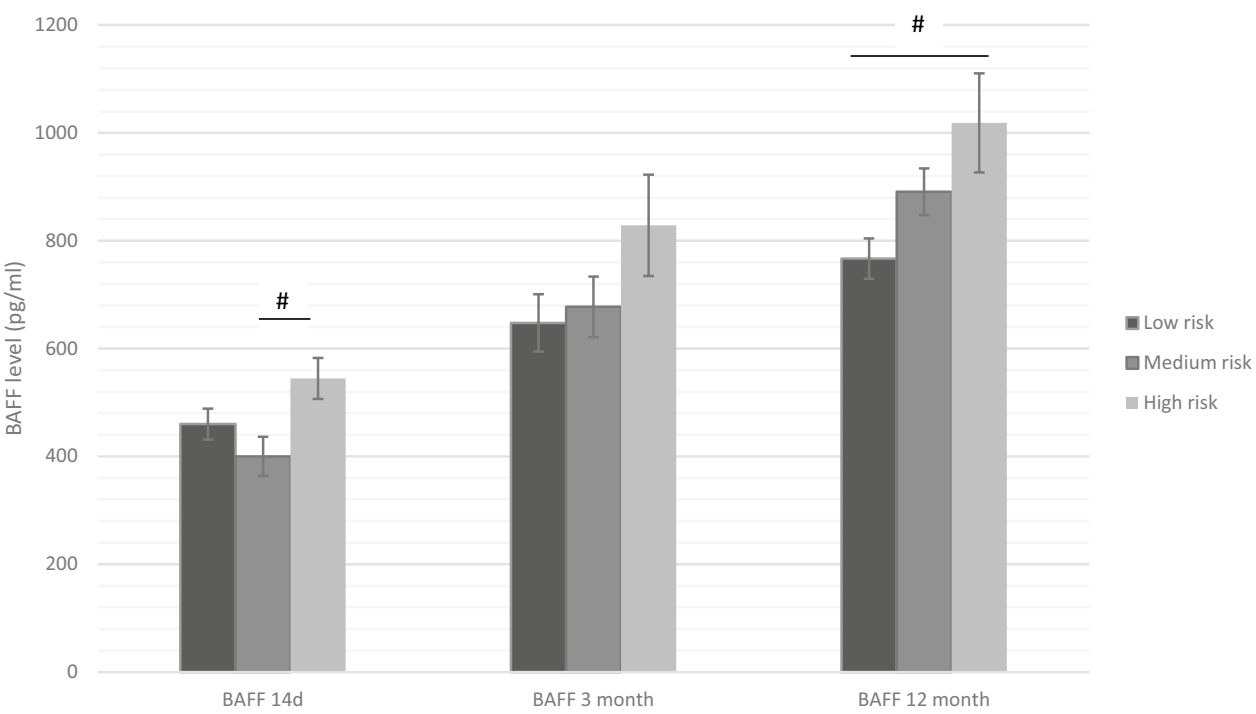

Also in comparison to the high-risk group, the mediumrisk group showed a trend towards an increased incidence of interstitial fibrosis and interstitial atrophy, reaching the level of significance in the biopsies, which were carried out between 31 and 100 days after the transplantation (ci: $p=0.02$; $c t: p=0.02$ ). If, on the other hand, one considers the occurrence of rejection at the mentioned time points, the group with medium risk showed no occurrence of $\mathrm{T}$ cellmediated (TCMR) and only one antibody-mediated rejection (AMR).

Compared to this, a total of 8 rejections were detected in the low-risk group (2 AMR, 6 TCMR). A total of 9 rejections were detected in the group of patients with a high immunological risk (2 AMR, 7 TCMR).

\section{De novo DSA development}

According to the initial stratification, only patients in the high-risk group had performed donor-specific antibodies. In the follow-up after transplantation, patients in the low- and medium-risk groups only developed HLA class II de novo DSA. It was found that $4.5 \%$ of the patients (2 von 44 ) in the low-risk group and $8.8 \%$ of the medium group (3 of 34) developed de novo DSA, in contrast, to the high-risk group with $22.7 \%$ dnDSA positive patients (10 of 44).

\section{Stratification according to BAFF median}

In a second step, the BAFF median at the time point 14 days of all patients $(n=122)$ was determined $(399.4 \mathrm{pg} / \mathrm{ml})$, and patients were stratified according to BAFF expression level: below vs. above BAFF median. Analysis of the baseline data showed that the donors of the cohort below the BAFF median were comparatively older (57 vs. 50 years, $\mathrm{p}=0.006$ ), whereas warm ischemia time was longer in patients above the BAFF median (14 min vs. $24 \mathrm{~min}$, $\mathrm{p}=0.03)$. All the other parameters showed no statistical difference.

The cohort above the BAFF median was more frequently induced with thymoglobulin ( 1.4 vs. $1.9, \mathrm{p}=0.007)$. Only one statistically significant difference could be observed in maintenance therapy, in the follow-up patients with $\mathrm{BAFF}$ expression levels above the median received higher steroid doses after one year. (2.8 vs. $4.4 \mathrm{mg}, \mathrm{p}=0.04$ ).

As in the first stratification, there was no relevant difference in resulting graft function (creatinine, eGFR, albuminuria) between the two groups during the observation period.

The histological analysis with regard to chronic lesions also showed no difference with the exception of focal sclerosis $(p=0.03)$ which was more intensified in the group above the median. In contrast, it was seen that patients with BAFF values above the median showed a marked trend towards more acute microvascular inflammation, in particular peritubular capillaritis $(p=0.07)$ and intimal arteritis $(p=0.09)$, all of which may be regarded as histopathological signs of AMR (Fig. 2 a and b). In contrast, lesions being associated with TCMR, e.g., interstitial inflammation and tubulitis differed not between both groups. When analyzing the biopsies for the occurrence of rejection, in accordance to the abovementioned observation, it was found that patients above the BAFF median suffered more often from rejections, especially antibody-mediated rejections (total: 6 vs. 12, ABMR: 1 vs. 4) (Table 3).

In significantly more patients in the group above the median BAFF, preTX DSA (18 (30.5\%) vs. $6(9.5 \%))$ could be detected. Seven patients $(11.1 \%)$ in the group below the median developed de novo DSA, whereas in the group above the median, 8 patients $(13.6 \%)$ were noticed with de novo DSA. Differences in the strength of 

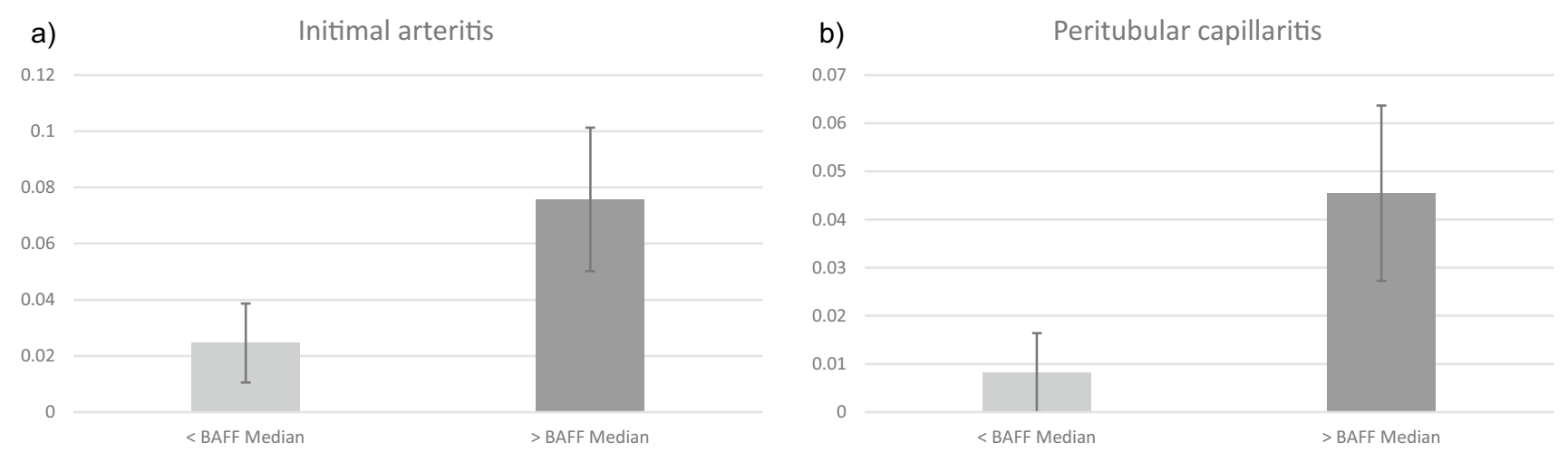

Fig. 2 a Difference according to BAFF median at 14 days related to the occurrence of intimal arteritis. b Difference according to BAFF median at 14 days related to the occurrence of peritubular capillaritis

the cumulative MFI values were not found in a significant range. However, at the time point of 12 months, there was a trend towards higher cumulative MFI values in the group above the median $(\mathrm{p}=0.09)$.

Additionally, patients with a combination of BAFF values above the median and simultaneous approval of any DSA (either preTx or dnDSA) showed a slight increase in any rejection episodes (either TCMR or AMR) compared to patients with low BAFF values and DSA (21.4 vs. $14.3 \%)$. Similar findings were made by comparing patients with high BAFF values and the presence of DSA compared to no DSA evidence (21.4 vs. 6.7\%).

\section{Discussion}

In our current study, BAFF correlates well with the immunological risk profile before transplantation. Patients with a low and medium immunological risk profile started with low BAFF values, whereas patients with a high immunological risk stand out from the start due to increased BAFF values. In the further course of time, however, especially patients with medium risk turned out with the most marked increase in BAFF expression level. At 12 month, this group almost approaches the BAFF values of the high group - keeping in mind that, according to our stratification, both groups receive the same doses of maintenance immunosuppression.

A study showed that pre-transplant BAFF levels represent the extent of pre-immunization of transplant candidates. In contrast, a connection between the post-transplant BAFF levels and the occurrence of rejections, donor-specific antibodies, or clinical outcome could no longer be found [17].

According to our initial stratification, only high-risk patients showed preTX donor-specific antibodies. In the follow-up, mainly patients with high risk developed de novo DSA. It was also striking that the other two groups rarely developed de novo DSA and, above all, no HLA class I antibodies were found. The development of donor-specific antibodies and the occurrence of antibody-mediated rejection was demonstrated in three patients. Looking at the stratification according to the BAFF median, more preTx DSA were found in the group with patients above the BAFF median. This group tended to develop more de novo DSA in the following time. Additionally, the combination "high BAFF + DSA" exposed patients for an increased risk of allograft rejection (21.4 vs. 14.3\% "low BAFF+DSA").

In literature, there are different observations regarding the relationship between BAFF and the development of DSA. In their work, Slavcec found no correlation between increased BAFF values and the increased occurrence of de novo donor-specific antibodies [18]. In contrast, in the work

Table 3 Incidence and type of rejection episodes according to BAFF median at 14 day

\begin{tabular}{|c|c|c|c|c|c|c|c|c|}
\hline & \multicolumn{4}{|c|}{ Below BAFF median } & \multicolumn{4}{|c|}{ Above BAFF median } \\
\hline & $\begin{array}{l}0-30 \text { days } \\
\text { postTx }\end{array}$ & $\begin{array}{l}30-100 \text { days } \\
\text { post Tx }\end{array}$ & $\begin{array}{l}\text { 100-365 days } \\
\text { postTx }\end{array}$ & $\begin{array}{l}>365 \text { days } \\
\text { post } T x\end{array}$ & $\begin{array}{l}0-30 \text { days } \\
\text { postTx }\end{array}$ & $\begin{array}{l}30-100 \text { days } \\
\text { postTx }\end{array}$ & $\begin{array}{l}\text { 100-365 days } \\
\text { postTx }\end{array}$ & $\begin{array}{l}>365 \text { days } \\
\text { postTx }\end{array}$ \\
\hline Rejection- total (n) & 6 & & & & 12 & & & \\
\hline TCMR (n) & 0 & 1 & 0 & 4 & 4 & 2 & 2 & 0 \\
\hline ABMR (n) & 1 & 0 & 0 & 0 & 3 & 0 & 1 & 0 \\
\hline
\end{tabular}

Abbreviations: TCMR, T cell-mediated rejection, $A B M R$ antibody-mediated rejection 
of Thibault et al., de novo DSA can be detected in transplant patients with high BAFF levels and unstable kidney function [9]. This observation can be partially understood in our work. Patients with stable function and high BAFF values were more likely to develop de novo DSA than patients with low BAFF levels.

When analyzing the rejection profile, patients with medium immunological risk showed the fewest rejection episodes. Two antibody-mediated rejections were detected in the high-risk group. However, according to the BAFF levels after 14 days, it was seen that high BAFF levels were associated with increased rejection rates, in particular antibody-mediated rejection. In line with this observation, this patient group also showed a trend towards more microvascular inflammation in the sense of peritubular capillaritis and intimal arteritis in the further analysis of the histological lesions score. Patients with rejections also had significantly higher BAFF values at 14 days and 3 months than patients without the presence of a rejection.

This finding is in agreement with some of the works that can be found in literature. Sango et al. were able to show that the detection of histological changes, which represent antibody-mediated rejection, is associated with increased BAFF levels [19]. In their work, Wang and colleagues were also able to find increased BAFF levels in patients with acute rejection compared to a collective with stable function after transplantation [20]. They observed both cohorts over a period of 6 months. In the work of Irure-Ventura et al., increased BAFF values before transplantation were associated with an increased occurrence of antibody-mediated rejection in the first 12 months [21]. In contrast to these examinations and the results of our cohort, the patients with ABMR in the work of Slavcec stood out due to the low BAFF level. The authors suspect that this is due to the increased binding of BAFF to its receptors [18]. In contrast, Snanoudj et al. found no connection between BAFF and the occurrence of a rejection [22].

$\mathrm{Xu}$ et al. found increased BAFF values in their work, especially in patients with impaired kidney function compared to patients with stable function [23]. We could not observe this in our collective, since all patients showed stable function regardless of the BAFF levels over time. However, it is important to mention that BAFF - only with the exception of the work by $\mathrm{Xu}$ et al. [23] — has not been used as a parameter to assess graft function so far. It is rather interesting in the context of characterizing patients changing immunological risk after transplantation and thereby ensuring that the usage of an appropriate maintenance immunosuppression prevents rejection episodes. Patients with a medium risk showed the greatest increase in their BAFF values over time (123\%) and nevertheless showed stable kidney function and few rejection episodes. A reduction in immunosuppression to the level of low-risk patients would therefore possibly be associated with an even greater increase in the BAFF values. This could result in a significantly higher risk of clinically relevant rejection episodes and a deterioration in graft function.

In our study, a significant change in BAFF levels in the group with medium immunological risk could be demonstrated. After 12 months, a risk profile similar to that of high-risk patients can be assumed. By adding the observation that patients with high BAFF values are at risk for an increased occurrence of microvascular inflammation and even severe rejections, BAFF can be used as an awareness factor. In this special patient population, the initial immunological risk allows a non $\mathrm{T}$ cell depleting induction therapy with Basiliximab as used in the low-risk group in comparison to thymoglobulin. Over time, however, an intensified maintenance immunosuppression, similar to those patients with a high risk, is indicated in order to balance the ongoing or aggravated immunological risk as reflected by changed BAFF values over time.

\section{Conclusion}

In this cohort of highly standardized kidney transplant recipients, BAFF reflects the clinically defined underlying immunological risk profile after kidney transplantation very well. Furthermore, higher BAFF values are associated with an intensified risk for rejection, especially antibody-mediated rejection. Patients with a medium immunological risk profile show a significant increase in BAFF values in the course after transplantation, so that a higher immunosuppressive therapy compared to low-risk patients is justified to relativize this changed immunological risk and BAFF can be used as an awareness factor for these patients over time.

Supplementary Information The online version contains supplementary material available at https://doi.org/10.1007/s12026-021-09205-4.

Acknowledgements We would like to thank Mrs. Stefanie Ellmann and Mrs. Alexandra Müller for their excellent technical assistance and Mrs. Lydia Walkowski for database management.

Author contribution Concept/design: SA, BB, BT. Data collection: SA, MN, BT.

Statistics: SA, BT.

Data analysis/interpretation: SA, MN, SL, BT.

Drafting article: SA, SL, BB, BT.

Critical revision of article: SA, MN, SL, BB, BT.

Approval of article: SA, MN, SL, BC, BB, BT.

Funding Open Access funding enabled and organized by Projekt DEAL. This work was funded by the Deutsche Forschungsgemeinschaft 
(DFG, German Research Foundation), project number 387509280, SFB 1350 to BT.

\section{Declarations}

Conflict of interest The authors of this manuscript have no conflict of interest to disclose as described by Immunological research.

Open Access This article is licensed under a Creative Commons Attribution 4.0 International License, which permits use, sharing, adaptation, distribution and reproduction in any medium or format, as long as you give appropriate credit to the original author(s) and the source, provide a link to the Creative Commons licence, and indicate if changes were made. The images or other third party material in this article are included in the article's Creative Commons licence, unless indicated otherwise in a credit line to the material. If material is not included in the article's Creative Commons licence and your intended use is not permitted by statutory regulation or exceeds the permitted use, you will need to obtain permission directly from the copyright holder. To view a copy of this licence, visit http://creativecommons.org/licenses/by/4.0/.

\section{References}

1. Chong AS, Sciammas R. Memory B cells in transplantation. Transplantation. 2015;99(1):21-8. https://doi.org/10.1097/TP. 0000000000000545.

2. Einecke G, Sis B, Reeve J, et al. Antibody-mediated microcirculation injury is the major cause of late kidney transplant failure. Am J Transplant. 2009;9(11):2520-31. https://doi.org/10.1111/j. 1600-6143.2009.02799.x.

3. Wiebe C, Gibson IW, Blydt-Hansen TD, et al. Evolution and clinical pathologic correlations of de novo donor-specific HLA antibody post kidney transplant. Am J Transplant. 2012;12(5):115767. https://doi.org/10.1111/j.1600-6143.2012.04013.x.

4. Vincent FB, Saulep-Easton D, Figgett WA, Fairfax KA, Mackay F. The BAFF/APRIL system: emerging functions beyond B cell biology and autoimmunity. Cytokine Growth Factor Rev. 2013;24(3):203-15. https://doi.org/10.1016/j.cytogfr.2013.04. 003 .

5. Tangye SG, Bryant VL, Cuss AK, Good KL. BAFF, APRIL and human B cell disorders. Semin Immunol. 2006;18(5):305-17. https://doi.org/10.1016/j.smim.2006.04.004.

6. Ng LG, Mackay CR, Mackay F. The BAFF/APRIL system: life beyond B lymphocytes. Mol Immunol. 2005;42(7):763-72. https://doi.org/10.1016/j.molimm.2004.06.041.

7. Mackay F, Tangye SG. The role of the BAFF/APRIL system in B cell homeostasis and lymphoid cancers. Curr Opin Pharmacol. 2004;4(4):347-54. https://doi.org/10.1016/j.coph.2004.02.009.

8. Lee WS, Amengual O. B cells targeting therapy in the management of systemic lupus erythematosus. Immunol Med. 2020;43(1):16-35. https://doi.org/10.1080/25785826.2019.16989 29.

9. Thibault-Espitia A, Foucher Y, Danger R, et al. BAFF and BAFF-R levels are associated with risk of long-term kidney graft dysfunction and development of donor-specific antibodies. Am J Transplant. 2012;12(10):2754-62. https://doi.org/10.1111/j. 1600-6143.2012.04194.x.

10. Schuster A, Jung B, Hofbauer J, et al. B-cell activating factor BAFF reflects patients' immunological risk profile after kidney transplantation. Transpl Immunol. 2017;45:35-41. https://doi.org/ 10.1016/j.trim.2017.08.006.

11. Pongpirul W, Chancharoenthana W, Pongpirul K, et al. B-cell activating factor (BAFF), a predictor of antibody mediated rejection in kidney transplantation recipients. Nephrology (Carlton). 2018;23(2):169-74. https://doi.org/10.1111/nep.12972.

12. Kühne L, Jung B, Poth $\mathrm{H}$, et al. Renal allograft rejection, lymphocyte infiltration, and de novo donor-specific antibodies in a novel model of non-adherence to immunosuppressive therapy. BMC Immunol. 2017;18(1):52. https://doi.org/10.1186/ s12865-017-0236-6.

13. Steines L, Poth H, Schuster A, et al. Anti-BAFF treatment interferes with humoral responses in a model of renal transplantation in rats. Transplantation. 2020;104(1):e16-22. https://doi.org/10. 1097/TP.0000000000002992.

14. Mujtaba MA, Komocsar WJ, Nantz E, et al. Effect of treatment with tabalumab, a B cell-activating factor inhibitor, on highly sensitized patients with end-stage renal disease awaiting transplantation. Am J Transplant. 2016;16(4):1266-75. https://doi.org/10. 1111/ajt.13557.

15 Banham GD, Flint S, Gibson A, et al. Belimumab in kidney transplantation. An experimental medicine, randomised, placebo-controlled phase 2 trial. Lancet. 2018;391(10140):2619-30. https:// doi.org/10.1016/S0140-6736(18)30984-X.

16. Roufosse C, Simmonds N, Clahsen-van Groningen M, et al. A 2018 Reference guide to the Banff classification of renal allograft pathology. Transplantation. 2018;102(11):1795-814. https://doi. org/10.1097/TP.0000000000002366.

17. Min JW, Kim KW, Kim B-M, et al. Clinical significance of preand post-transplant BAFF levels in kidney transplant recipients. PLoS ONE. 2016;11(9):e0162964. https://doi.org/10.1371/journ al.pone.0162964.

18. Slavcev A, Brozova J, Slatinska J, et al. Soluble BAFF cytokine levels and antibody-mediated rejection of the kidney allograft. Arch Immunol Ther Exp (Warsz ). 2016;64(Suppl 1):47-53. https://doi.org/10.1007/s00005-016-0428-4.

19. Sango C, Merino D, San Segundo D, et al. B-cell-activating factor levels are associated with antibody-mediated histological damage in kidney transplantation. Transplant Proc. 2016;48(9):2910-2. https://doi.org/10.1016/j.transproceed.2016.09.019.

20. Wang X-Z, Wan Z, Xue W-J, Zheng J, Li Y, Ding CG. B-cell activating factor predicts acute rejection risk in kidney transplant recipients: a 6-month follow-up study. Front Immunol. 2019;10:1046. https://doi.org/10.3389/fimmu.2019.01046.

21. Irure-Ventura J, San Segundo D, Rodrigo E, et al. High Pretransplant BAFF levels and B-cell subset polarized towards a memory phenotype as predictive biomarkers for antibody-mediated rejection. Int J Mol Sci. 2020;21(3). https://doi.org/10.3390/ijms2 1030779.

22. Snanoudj R, Candon S, Roelen DL, et al. Peripheral B-cell phenotype and BAFF levels are associated with HLA immunization in patients awaiting kidney transplantation. Transplantation. 2014;97(9):917-24. https://doi.org/10.1097/01.TP.0000438211. $34842.5 \mathrm{e}$.

23. Xu H, He X, Liu Q, et al. Abnormal high expression of B-cell activating factor belonging to the TNF superfamily (BAFF) associated with long-term outcome in kidney transplant recipients. Transplant Proc. 2009;41(5):1552-6. https://doi.org/10.1016/j. transproceed.2008.10.024.

Publisher's note Springer Nature remains neutral with regard to jurisdictional claims in published maps and institutional affiliations. 